\title{
THEOREMS ON CARTAN SUBALGEBRAS LIKE SOME ON CARTER SUBGROUPS
}

\author{
BY \\ ERNEST L. STITZINGER
}

\begin{abstract}
We consider some results on the Cartan subalgebras of a solvable Lie algebra which are analogues to some results on Carter subgroups of a finite solvable group. Only solvable Lie algebras are considered here.
\end{abstract}

Much attention has recently been given to the Carter subgroups in finite solvable groups. Since the Carter subgroups are analogues of the Cartan subalgebras of a Lie algebra, it would seem to be of interest to see which of the results on the former are shared by the latter. It has been shown in various places (see for instance [10]) that Cartan subalgebras exist in any solvable Lie algebra. Under certain conditions it has been shown that all Cartan subalgebras of a solvable Lie algebra are conjugate (see [2] and [10]). However, in general this result fails; in fact, there exist solvable Lie algebras whose Cartan subalgebras are of different dimensions [10]. The present paper continues the investigation into which properties of Carter subgroups are shared by Cartan subalgebras. At times a result and its proof carry over virtually unchanged while at other times this is not the case. All Lie algebras considered here are solvable and finite dimensional. The nil-radical of a Lie algebra $L$ will be denoted by $N(L) . L$ is said to be of nilpotent length $t$ if $t$ is the smallest natural number such that there exists a chain of ideals of $L$,

$$
0=L_{0} \subset L_{1} \subset \cdots \subset L_{t}=L,
$$

such that each factor is nilpotent.

We begin by recording the following characterization of Cartan subalgebras which is certainly widely known.

LEMMA 1. Let $H$ be a nilpotent subalgebra of the Lie algebra L. The following are equivalent:

(1) $H$ is a Cartan subalgebra of $L$,

(2) if $K$ is a subalgebra of $L, H \subseteq K \subseteq L$, then $K$ is its own normalizer in $L$, and

(3) every maximal chain joining $H$ to $L$ has the property that any member of the chain is self-normalizing in the next.

Received by the editors September 14, 1970.

AMS 1969 subject classifications. Primary 1730.

Key words and phrases. Cartan subalgebra.

Copyright (C) 1971, American Mathematical Society 
Proof. That (1) implies (2) is shown in Theorem 2 of [2] and (2) obviously implies (3). Assume that (3) holds and let $x$ be in the normalizer of $H$ but not in $H$. Put $K=\langle x, H\rangle$. Then $H \subset K$ can be completed to a maximal chain joining $H$ to $L$ so that $H$ must be self-normalizing in $K$ which is not the case.

We would like to consider the relationship of Cartan subalgebras to the Lie algebra analogue of system normalizers of P. Hall. With the apparent lack of any suitable Lie algebra analogue to Sylow systems, we propose to consider the Lie algebra counterpart to the abstract definition of system normalizers as given in [8]. Let $L$ be a solvable Lie algebra and let $M(L)$ be the set of all subalgebras $A$ of $L$ with the following properties:

(1) $A$ can be joined to $L$ by a chain of subalgebras of $L$ each maximal and selfnormalizing in the next.

(2) No maximal subalgebra of $A$ is self-normalizing in $A$.

Since a Lie algebra is nilpotent if and only if all its maximal subalgebras are ideals [1], $M(L)$ consists precisely of those subalgebras of $L$ which are nilpotent and satisfy (1). The minimal members of $M(L)$ are a Lie algebra counterpart to system normalizers of finite solvable groups. By Lemma 1, the Cartan subalgebras of $L$ are contained in $M(L)$ and hence each Cartan subalgebra contains a minimal member of $M(L)$.

Lemma 2. Let $L$ be a Lie algebra, $N$ be an ideal of $L$ and $D \in M(L)$. Then $D+N / N \in M(L / N)$.

Proof. Straightforward.

Carter has shown [4, Theorem 5.6] that in groups of nilpotent length 2 or less, the Carter subgroups and the system normalizers coincide. The analogue of this result holds in Lie algebras; that is, in a Lie algebra $L$ of nilpotent length 2 or less the Cartan subalgebras coincide with the minimal members of $M(L)$. We show in fact the following result which only appears to be more general.

THEOREM 1. Let L be a Lie algebra of nilpotent length 2 or less. Then the Cartan subalgebras of $L$ are precisely those subalgebras in $M(L)$.

Proof. If $L$ is nilpotent, then the result is trivial. We let $L$ be of nilpotent length 2 and proceed by induction on the dimension of $L$. Let $D \in M(L)$ and let $D=D_{0} \subset \ldots \subset D_{t} \subset L$ be a chain of subalgebras of $L$ each maximal and selfnormalizing in the next. If $D$ is maximal in $L$, then the result is immediate. If $D$ is not maximal in $L$, then $D$ is a Cartan subalgebra of $D_{t}$ by induction. $D_{t}$ is selfnormalizing in $L$ and $L$ has nilpotent length 2, hence $L=D_{t}+N(L)$. Now $D_{t} \cap N(L)$ is an ideal in $D_{t}$ and $D_{t} \cap N(L)$ is not self-normalizing in $N(L)$ since $N(L)$ is nilpotent and $D_{t} \neq N(L)$. Then, since $D_{t}$ is maximal and the $L$-normalizer of $D_{t} \cap N(L)$ contains $D_{t}$ properly, $D_{t} \cap N(L)$ is an ideal of $L$. Then $L / N(L)$ $\simeq D_{t} / D_{t} \cap N(L)$ is nilpotent and hence $D_{t} / D_{t} \cap N(L)$ is a Cartan subalgebra of $L / D_{t} \cap N(L)$. Consequently $D$ is a Cartan subalgebra of $L$ by Lemma 4 of [2]. 
Therefore each element of $M(L)$ is a Cartan subalgebra of $L$ and since each Cartan subalgebra of $L$ is contained in $M(L)$, the result holds.

In groups of nilpotent length three or less, Carter has shown in Theorem 2 of [6] that any system normalizer must be contained in precisely one Carter subgroup. The analogous result holds for Lie algebras; that is, in a Lie algebra $L$ of nilpotent length 3 or less each minimal member of $M(L)$ is contained in precisely one Cartan subalgebra. We can show this result in slightly more general form.

THEOREM 2. Let $L$ be of nilpotent length 3 or less. Then each element of $M(L)$ is contained in precisely one Cartan subalgebra of $L$.

Proof. If $L$ is of nilpotent length 2 or less, then the result holds. Therefore let $L$ have nilpotent length 3 and proceed by induction on the dimension of $L$. Let $D \in M(L)$. Then $D+N(L) / N(L)$ is a Cartan subalgebra of $L / N(L)$ by Lemma 2 and Theorem 1. Let $E$ be a Cartan subalgebra of $D+N(L)$. Then by Lemma 4 of [2], $E$ is a Cartan subalgebra of $L$ and clearly $E+N(L)=D+N(L)$. Let $S=E+N(L)$. For $x \in D$, let $S_{x}$ be the Fitting null component of ad $x$ acting on $S$ and let

$$
K=\bigcap_{x \in D} S_{x}
$$

Since $D$ is nilpotent, $D \subseteq K$. If $y \notin K$, then $y \notin S_{x}$ for some $x \in D$ and $[y, x] \notin S_{x}$. Hence $K$ is self-normalizing in $S$. Furthermore,

$$
K=K \cap(D+N(L))=D+(K \cap N(L)) .
$$

Since the inner derivation of each element of $D$ is nilpotent acting on $K$, since $K \cap N(L)$ is an ideal of $K$ and since $K \cap N(L)$ is nilpotent, using Engel's Theorem, $K$ is nilpotent. Therefore $K$ is a Cartan subalgebra of $L$. Therefore each element of $M(L)$ is contained in a Cartan subalgebra of $L$.

Suppose that $D$ is contained in Cartan subalgebras $E_{1}$ and $E_{2}$ of $L$. Then $E_{1}+N(L) / N(L)=D+N(L) / N(L)=E_{2}+N(L) / N(L)$, hence $E_{1}+N(L)=D+N(L)$ $=E_{2}+N(L)$. Then $E_{1}=E_{1} \cap(N(L)+D)=\left(E_{1} \cap N(L)\right)+D$ and $E_{2}=\left(E_{2} \cap N(L)\right)$ $+D$. Let $A$ be a minimal ideal of $L$. By induction $A+E_{1}=A+E_{2}$. Now $E_{1}$ and $E_{2}$ are both Cartan subalgebras of $A+E_{1}$ and $A+E_{1}$ evidently has the property that the intersection of the members of the lower central series of $A+E_{1}$ is abelian. So by a discussion on p. 117 of [10], $E_{1}$ and $E_{2}$ must be conjugate under $I+\operatorname{ad} a$ for some $a \in A$. Also $A$, as a minimal ideal of $L$ must be in the center of $N(L)$, hence

$$
\begin{aligned}
E_{1} \cap N(L) & =E_{2}(I+\operatorname{ad} a) \cap N(L) \\
& =\left(E_{2} \cap N(L)\right)(I+\operatorname{ad} a)=E_{2} \cap N(L) .
\end{aligned}
$$

Then $E_{1}=\left(E_{1} \cap N(L)\right)+D=\left(E_{2} \cap N(L)\right)+D=E_{2}$.

We shall find a characterization of Cartan subalgebras by strengthening (1) in the definition of $M(L)$. If $A$ and $B$ are subalgebras of $L$ and $B$ is maximal and selfnormalizing in $A$, then let $\operatorname{core}_{A}(B)$ be the maximal ideal of $A$ which is contained 
in $B$. Let $L$ be a solvable Lie algebra and let $\bar{M}(L)$ be the set of all subalgebras $A$ of $L$ with the following properties:

(1) There exists a chain $A=A_{0} \subset \ldots \subset A_{n}=L$ of subalgebras of $L$ each maximal and self-normalizing in the next.

(2) $A_{i} /$ core $_{A_{i+1}}\left(A_{i}\right)$ is nilpotent.

(3) There does not exist a subalgebra $K$ of $A$ such that $K$ is maximal and selfnormalizing in $A$ and $K / \operatorname{core}_{A}(K)$ is nilpotent.

Lemma 3. Let $L$ be a nonnilpotent, solvable Lie algebra and let $C$ be a Cartan subalgebra of $L$. Then $L$ contains a self-normalizing maximal subalgebra $M$ which contains $C$ and $M / \operatorname{core}_{L}(M)$ is nilpotent.

Proof. Let $K$ be the intersection of the members of the lower central series of $L$ and let $G$ be a proper subalgebra of $K$ which is an ideal of $L$ and is maximal with these properties. Now $(C+G) / G$ is a Cartan subalgebra of $L / G$ and there exists a maximal self-normalizing subalgebra $M / G$ of $L / G$ which contains $(C+G) / G$ by Lemma 1 . Obviously $M / G$ does not contain $K / G$ and hence $M / G+K / G=L / G$. Furthermore $M / G \cap K / G$ is an ideal in $M / G$ and since $K / G$ is abelian, $M / G \cap K / G$ is an ideal in $K / G$. Hence $M / G \cap K / G$ is an ideal in $L / G$ and $G \subseteq M \cap K \subset K$, hence $M \cap K=G$. Then $M / G=M / M \cap K=M+K / K=L / K$ which is nilpotent. Then $M / \operatorname{core}_{L}(M)$ is nilpotent. Since $C \subseteq M$, the result follows.

TheOREM 3. Let $L$ be a solvable Lie algebra and let $A$ be a subalgebra of $L$. Then $A$ is a Cartan subalgebra of $L$ if and only if $A \in \bar{M}(L)$.

Proof. Suppose $A \in \bar{M}(L)$. By Lemma 3, $A$ must be nilpotent. Then since $A$ is self-normalizing in $A_{1}, A$ is a Cartan subalgebra of $A_{1}$. Since $A_{1} /$ core $_{A_{2}}\left(A_{1}\right)$ is a Cartan subalgebra of $A_{2} / \operatorname{core}_{A_{2}}\left(A_{1}\right), A$ is a Cartan subalgebra of $A_{2}$. Evidently we may continue this argument to show that $A$ is a Cartan subalgebra of $L$.

On the other hand, if $A$ is a Cartan subalgebra of $L$ and $L$ is not nilpotent, then by Lemma 3 there exists a maximal subalgebra $M$ of $L$ which contains $A$ and $M / \operatorname{core}_{L}(M)$ is nilpotent. $A$ is a Cartan subalgebra of $M$ so that if $M$ is not nilpotent then there exists a maximal subalgebra $N$ or $M$ which contains $A$ and $N /$ core $_{M}(N)$ is nilpotent. This may be repeated until we reach a nilpotent subalgebra $K$ which contains $A$ and $A$ is a Cartan subalgebra of $K$. Then $A=K$ and the result holds.

We now turn in another direction. If $H$ is a subalgebra of $L$ it is not true in general that any Cartan subalgebra of $H$ is the intersection of $H$ with a Cartan subalgebra of $L$. However, we find in the next result a case when this is true.

THEOREM 4. Let $H$ be a subalgebra of $L$ such that $L=N(L)+H$. Then every Cartan subalgebra $K$ of $H$ is of the form $H \cap C$ where $C$ is a Cartan subalgebra of $L$ and $C$ contains the $L$-normalizer of $K$. If $D$ is any Cartan subalgebra of $L$ which contains $K$, then $D=C$. 
Proof. Let $K$ be a Cartan subalgebra of $H$. For $x \in K$, let $L_{x}$ be the Fitting null component of ad $x$ acting on $L$ and let $S=\bigcap_{x \in K} L_{x}$. Since $K$ is nilpotent, $K \subseteq S$. We claim that $S$ is a Cartan subalgebra of $L$ and that $S \cap H=K$. First, if $y \notin S$, then there exists $x \in K$ such that $y \notin L_{x}$ and then $[x, y] \notin L_{x}$. Thus $[x, y] \notin S$ so that $y$ is not in the normalizer of $S$; that is, $S$ is its own normalizer in $L$. Now $K \subseteq S \cap H$ so suppose this inclusion is proper. Each element of $K$ induces a nilpotent linear transformation on the vector space quotient $(S \cap H) / K$, hence by Engel's Theorem, there exists a one-dimensional subspace of $(S \cap H) / K$ which is annihilated by each of these linear transformations. This is equivalent to saying that there exists an element of $S \cap H$ which is not in $K$ but is in the normalizer of $K$, an impossibility since $K$ is self-normalizing in $H$. Hence $S \cap H=K$.

We claim next that $K+N(L)=S+N(L)$. Evidently $K+N(L) \subseteq S+N(L)$ so suppose the containment is proper. Then every element in $K+N(L)$ induces a nilpotent linear transformation on $(S+N(L)) /(K+N(L))$. Again by Engel's Theorem there exists a one-dimensional subspace of $(S+N(L)) /(K+N(L))$ which is annihilated by all of these linear transformations. This is equivalent to saying that there exists an element of $S+N(L)$ not in $K+N(L)$ which normalizes $K+N(L)$ which is impossible since $(K+N(L)) / N(L)$ is a Cartan subalgebra of $(H+N(L)) / N(L)=L / N(L)$ and so $(K+N(L)) / N(L)$ is self-normalizing in $S+N(L) / N(L)$. Therefore $K+N(L)=S+N(L)$ and

$$
S=S \cap(K+N(L))=K+(S \cap N(L)) .
$$

Now $S$ is nilpotent. For $S \cap N(L)$ is a nilpotent ideal in $S$ and each element of $K$ induces a nilpotent linear transformation on $S$, hence, using Engel's Theorem, $S$ is nilpotent. Furthermore by the way $S$ is defined the $L$-normalizer of $K$ is contained in $S$.

It remains to show that $S$ is the only Cartan subalgebra of $L$ which contains $K$. We use induction on the dimension of $L$. Suppose that $F$ is another Cartan subalgebra of $L$ which contains $K$ and let $N$ be a minimal ideal of $L$. Then $K+N / N$ is contained in $S+N / N$ and $F+N / N$, so, by induction, $S+N=F+N$. Suppose $S+N \subset L$. Since $S=K+(S \cap N(L)), S+N=K+(S \cap N(L))+N$ where $S \cap N(L)$ and $N$ are both nilpotent ideals of $S+N$. Hence $S+N$ is the sum of $K$ and the nilradical of $S+N, K$ is a Cartan subalgebra of itself, and $K$ is contained in $S$ and $F$. Since $S$ and $F$ are both Cartan subalgebras of $S+N, S=F$ by induction.

We may now suppose that $S+N=L$. Since $N$ is an abelian ideal and $S$ is nilpotent, there exists $x \in N$ such that $S(I+\operatorname{ad} x)=F$. Since $N$ is a minimal ideal in $L$, either $N \subseteq S$ or $N \cap S=0$. In the first case $L$ is nilpotent and $S=F$. Suppose that $N \cap S=0$. Since $S(I+\operatorname{ad} x)=F$ for some $x \in N, N \cap F=0$. Then $K=K+(N \cap S)$ $=(K+N) \cap S$ and $K=K+(N \cap F)=(K+N) \cap F$. Since $x \in N, I+$ ad $x$ leaves $K+N$ invariant. Then $K(I+\operatorname{ad} x)=((K+N) \cap S)(I+\operatorname{ad} x)=(K+N)(I+\operatorname{ad} x)$ $\cap S(I+\operatorname{ad} x)=(K+N) \cap F=K$. Hence $I+\operatorname{ad} x$ leaves $K$ invariant. This forces $x$ to be in the $L$-normalizer of $K$, hence $x \in S$. Then $F=S(I+\operatorname{ad} x)=S$. 
COROLlary. Let $H$ be a nilpotent subalgebra of $L$ such that $L=H+N(L)$. Then there exists a unique Cartan subalgebra $C$ of $L$ which contains $H$. $C$ also contains the normalizer of $H$.

We can use this corollary to find a characterization of Cartan subalgebras.

Theorem 5. (1) Let $K$ be a Cartan subalgebra of $L$ and $N$ be an ideal of $L$. Then $K+N / N$ is a Cartan subalgebra of $L / N$, and of $H$ is a subalgebra of $L$ containing $K$ and $\mathrm{H}+\mathrm{N} / \mathrm{N}$ is nilpotent, then $\mathrm{H}+\mathrm{N}=\mathrm{K}+\mathrm{N}$.

(2) Let $0=N_{0} \subset \ldots \subset N_{r}=L$ be a chain of subalgebras of $L$ such that each is an ideal in the next and $N_{i+1} / N_{i}$ is nilpotent. Let $K \subseteq L$. If for each $i, K+N_{i} / N_{i}$ is nilpotent and $K+N_{i} \mid N_{i}$ is contained properly in no nilpotent subalgebra of $L / N_{i}$, then $K$ is a Cartan subalgebra of $L$.

Proof. (1) This follows from Lemma 3 of [2] and the definition of Cartan subalgebras.

(2) Use induction on $r$. If $r=1$, it is immediate. Now $K+N_{1} / N_{1}$ satisfies the condition of the theorem in $L / N_{1}$, hence $K+N_{1} / N_{1}$ is a Cartan subalgebra of $L / N_{1}$. Furthermore $K=K+N_{0} / N_{0}$ is nilpotent by hypothesis. Also $K+N_{1}$ is the sum of $K$ and the nil-radical of $K+N_{1}$ so that by the Corollary $K$ is contained in a Cartan subalgebra $C$ of $K+N_{1}$. Now $C=C+N_{0} / N_{0}$ is nilpotent, hence by hypothesis $C=K$. Hence $K$ is a Cartan subalgebra of $K+N_{\mathrm{i}}$ so that, by Lemma 4 of [2], $K$ must be a Cartan subalgebra of $L$.

We now turn to the Lie algebra analogue of the cover-avoidance property possessed by Carter subgroups. We need the following terminology. Let $\boldsymbol{H}$ be a subalgebra of $L$. Let $M$ and $N$ be subalgebras of $L$ such that $N \subset M,[N, M] \subseteq N$, $[H, M] \subseteq M$ and $[H, N] \subseteq N$. Then $M / N$ is called an $H$-factor of $L$. If there exist no subalgebras of $L$ contained between $M$ and $N$ which are $H$-invariant, then $M / N$ is called an irreducible $H$-factor. A chain $0=L_{0} \subset \ldots \subset L_{n}=L$ in which each adjacent pair is an irreducible $H$-factor will be called an $H$-chain. If $[M, H] \subseteq N$, then $M / N$ is called a central $H$-factor and in the contrary case, $M / N$ is called an eccentric $H$-factor. Evidently irreducible $H$-factors are either central or $[M, H]=M$. The subalgebra $N+(M \cap H)$ of $L$ lies between $N$ and $M$. We say that $H$ avoids $M / N$ if $N+(M \cap H)=N$ and covers $M / N$ if $N+(M \cap H)=M$. Clearly $H$ is in the normalizer of $N+(M \cap H)$. Using the analogous terminology for groups, Carters cover-avoidance theorem shown in [5] is as follows: A Carter subgroup $H$ of the solvable group $G$ covers all central irreducible $H$-factors and avoids all eccentric irreducible $H$-factors. Before proceeding to the Lie algebra case we need the following lemma.

LEMMA 4. Let $H$ be a nilpotent subalgebra of $L$ and let $M / N$ be an irreducible $H$-factor of L. Then $M+H / N$ is nilpotent if and only if $M / N$ is $H$-central.

Proof. Suppose $M / N$ is $H$-central and note that $M+H / N$ is the sum of $M / N$ and $N+H / N$. Since $H$ is nilpotent, $N+H / N$ is nilpotent. Since $M / N$ is an irre- 
ducible $H$-factor, $M / N$ is abelian. Since $H$ is in the normalizer of $M, M / N$ is an ideal of $M+H / N$. Since $M / N$ is $H$-central, $N+H / N$ is an ideal of $M+H / N$. Hence $M+H / N$ is the sum of two nilpotent ideals and is therefore nilpotent. If $M+H / N$ is nilpotent, then since $M / N$ is a minimal ideal in $M+H / N, M / N$ is contained in the center of $M+H / N$. Hence $[M, H] \subseteq N$ and $M / N$ is $H$-central.

THEOREM 6. Let $H$ be a Cartan subalgebra of $L$. Then $H$ covers all central irreducible $H$-factors and avoids all eccentric irreducible $H$-factors.

Proof. Let $M / N$ be an irreducible $H$-factor. Then either $N+(M \cap H)=N$ or $N+(M \cap H)=M$; that is, either $H$ covers $M / N$ or $H$ avoids $M / N$.

Suppose $H$ avoids $M / N$ and that $M / N$ is $H$-central. Then by Lemma $4, M+H / N$ is nilpotent. Now $(N+H) / N$ is a Cartan subalgebra of $L / N$ and so is a Cartan subalgebra of $M+H / N$. But $M+H / N$ is nilpotent, hence $M+H=N+H$. On the other hand, $H$ avoids $M / N$, hence $N+(H \cap M)=N$ and $H \cap M \subseteq N$ so that $H \cap M=H \cap N$. Now $M$ and $N$ being $H$-invariant, $H \cap M=H \cap N$ and $H+M$ $=H+N$ imply $M=N$, a contradiction.

Suppose now that $H$ covers $M / N$ and that $M / N$ is $H$-eccentric. By Lemma 4 $M+H / N$ is not nilpotent. On the other hand, since $H$ covers $M / N, N+(M \cap H)$ $=M$, consequently $H+M=H+N+(H \cap M)=H+N$. Then $M+H / N=N+H / N$ $\simeq H / H \cap N$, the latter being nilpotent. This is a contradiction.

Bauman, in the more general setting of formation theory, has found this covering and avoidance property actually characterizes Carter subgroups. The analogous property in Lie algebras is the content of

THEOREM 7. Let $L$ be a solvable Lie algebra and let $\left\{L_{i}\right\}$ be an $H$-chain of $L$ such that $H$ covers $L_{i} / L_{i-1}$ if and only if $L_{i} / L_{i-1}$ is $H$-central. Then $H$ is a Cartan subalgebra of $L$.

Proof. Considering the chain $\left\{L_{i} \cap H\right\}$ one sees that $H$ is nilpotent. By Lemma 4, $L_{i} / L_{i-1}$ is $H$-central if and only if $L_{i}+H / L_{i-1}$ is nilpotent. We therefore use the hypothesis that $H$ covers $L_{i} / L_{i-1}$ if and only if $L_{i}+H / L_{i-1}$ is nilpotent. We use induction on $\operatorname{dim} L$ and note that the result is trivial if $\operatorname{dim} L=1$. Assume $\operatorname{dim} L>1$. If $H=L$, then $H$ is a Cartan subalgebra of $L$. If $H \subset L$, then $H$ does not cover all the factors of $\left\{L_{i}\right\}$. Choose $\tau$ minimal so that $H$ covers all factors from $L_{\tau}$ to $L$. Then since $H$ covers all factors from $L_{\tau}$ to $L, L=H+L_{\tau}$. Since $L_{\tau}$ is $H$-invariant, $L_{\tau}$ is an ideal of $L$ and since $L_{\tau-1}$ is $L_{\tau}$ and $H$ invariant, $L_{\tau-1}$ is an ideal of $L$. Furthermore $L / L_{\tau}$ is nilpotent and since $H$ avoids $L_{\tau} / L_{\tau-1}, L / L_{\tau-1}$ is not nilpotent. Furthermore $L_{\tau-1}+H \subset L$, for if not then $L_{\tau-1}+\left(H \cap L_{\tau}\right)=\left(L_{\tau-1}+H\right) \cap L_{\tau}=L \cap L_{\tau}=L_{\tau}$ which contradicts $H$ avoiding $L_{\tau} / L_{\tau-1}$. In $H+L_{\tau-1}$ form the $H$-invariant chain $\left\{L_{i} \cap\left(H+L_{\tau-1}\right)\right\}$. This chain satisfies the hypothesis of the theorem. In $L / L_{\tau-1}$ the $H+L_{\tau-1} / L_{\tau-1}$ invariant chain of homomorphic images of $\left\{L_{i}\right\}$ also satisfies the hypothesis of the theorem. Assume $\operatorname{dim} L_{\tau-1} \neq 0$. Then by induction $H$ is a Cartan subalgebra in $H+L_{\tau-1}$ and $H+L_{\tau-1} / L_{\tau-1}$ is a Cartan subalgebra in $L / L_{\tau-1}$. 
Hence $H$ is a Cartan subalgebra in $L$ by Lemma 4 of [2]. Assume $\operatorname{dim} L_{\tau-1}=0$. Then $L_{\tau}$ is a minimal ideal and $H$ is a maximal subalgebra which complements $L_{\tau}$. Then $H \simeq L / L_{\tau}$ is nilpotent and since $H$ is a nilpotent maximal subalgebra which complements a minimal ideal in a nonnilpotent Lie algebra, $H$ is self-normalizing and is therefore a Cartan subalgebra of $L$.

We now turn our attention to a Lie algebra counterpart of a splitting property shown in [11]. Let $L_{\infty}^{1}$ be the intersection of the lower central series of $L$ and for $i \geqq 1$ let $L_{\infty}^{i}$ be the intersection of the lower central series of $L_{\infty}^{i-1}$. Since $L$ is solvable $L_{\infty}^{i}=0$ for some $i$. We use analogous notation for the solvable group case. Carter has shown in [11] that if $G$ is a finite solvable group and if, for some $i \geqq 1, G_{\infty}^{i}$ is abelian but not the identity of $G$, then $G_{\infty}^{i}$ is complemented in $G$ by the relative system normalizers of a Sylow system of $G_{\infty}^{i-1}$, all complements of $G_{\infty}^{i}$ arise this way and that all complements of $G_{\infty}^{i}$ are conjugate. In the Lie algebra case it is known (see p. 117 of [10]) that if $L_{\infty}^{1}$ is abelian, then $L_{\infty}^{1}$ is complemented by precisely the Cartan subalgebras of $L$ and all such complements are conjugate under some $I+\operatorname{ad} a, a \in L_{\infty}^{1}$. We find a result which generalizes this and is somewhat analogous to Carter's result.

THEOREM 8. Suppose that $L_{\infty}^{n}$ is abelian but not 0 for some $n \geqq 1$ and let $H$ be a Cartan subalgebra of $L_{\infty}^{n-1}$. Let $H_{0}$ be the Fitting null component of ad $H$ acting on $L$. Then $H_{0}$ is a complement of $L_{\infty}^{n}$ in $L$. Furthermore if $K$ is a complement of $L_{\infty}^{n}$ in $L$, then $K$ is the Fitting null component of ad $J$ acting on $L$ where $J$ is a Cartan subalgebra of $L_{\infty}^{n-1}$. All complements of $L_{\infty}^{n}$ in $L$ are conjugate by automorphisms of $L$ of the form $I+\operatorname{ad} x, x \in L_{\infty}^{n}$.

Proof. If $n=1$ the result follows from the discussion on p. 117 of [10]. Let $n \geqq 2$. Then $L_{\infty}^{n-1}=H+L_{\infty}^{n}$ where $H$ is a Cartan subalgebra of $L_{\infty}^{n-1}$, all complements of $L_{\infty}^{n}$ in $L_{\infty}^{n-1}$ are Cartan subalgebras of $L_{\infty}^{n-1}$ and they are all conjugate under automorphisms of $L_{\infty}^{n-1}$ of the form $I+$ ad $x, x \in L_{\infty}^{n}$. Let $H_{0}$ and $H_{1}$ be the Fitting null and one component of ad $H$ acting on $L$. Since $H \subseteq L_{\infty}^{n-1}$ and $L_{\infty}^{n-1}$ is an ideal of $L$, $H_{1} \subseteq L_{\infty}^{n-1}$. Then since $L_{\infty}^{n}$ is the Fitting one component of ad $H$ acting on $L_{\infty}^{n-1}$, $H_{1}=L_{\infty}^{n}$. Hence $L_{\infty}^{n}$ is complemented in $L$ by $H_{0}$.

Let $K$ be an arbitrary complement of $L_{\infty}^{n}$ in $L$. Then $L_{\infty}^{n-1}=L_{\infty}^{n-1} \cap\left(K+L_{\infty}^{n}\right)$ $=\left(L_{\infty}^{n-1} \cap K\right)+L_{\infty}^{n}$ and $L_{\infty}^{n} \cap\left(L_{\infty}^{n-1} \cap K\right)=0$, hence $L_{\infty}^{n-1} \cap K$ is a complement of $L_{\infty}^{n}$ in $L_{\infty}^{n-1}$ and so must be a Cartan subalgebra of $L_{\infty}^{n-1}$. Furthermore $L_{\infty}^{n-1} \cap K$ is a nilpotent ideal in $K$, hence $K$ is the Fitting null component of ad $\left(L_{\infty}^{n-1} \cap K\right)$ acting on $K$. Since $K$ is a complement of $L_{\infty}^{n}$ and $L_{\infty}^{n}$ is the Fitting one component of ad $\left(L_{\infty}^{n-1} \cap K\right)$ acting on $L_{\infty}^{n-1}, K$ is the Fitting null component of ad $\left(L_{\infty}^{n-1} \cap K\right)$ acting on $L$. Therefore, combining this with the result of the previous paragraph, the complements of $L_{\infty}^{n}$ in $L$ are precisely the Fitting null components of the Cartan subalgebras of $L_{\infty}^{n-1}$ acting on $L$. Evidently each Cartan subalgebra of $L_{\infty}^{n-1}$ is contained in precisely one complement of $L_{\infty}^{n}$ in $L$ and each complement of $L_{\infty}^{n}$ in $L$ contains exactly one Cartan subalgebra of $L_{\infty}^{n-1}$. 
It remains to show the conjugacy part of the theorem. Let $K_{1}$ and $K_{2}$ be two complements of $L_{\infty}^{n}$ and let $H_{1}=K_{1} \cap L_{\infty}^{n-1}$ and $H_{2}=K_{2} \cap L_{\infty}^{n-1}$. As we have seen $H_{1}$ and $H_{2}$ are Cartan subalgebras of $L_{\infty}^{n-1}$ and these are conjugate in $L_{\infty}^{n-1}$ under $I+\operatorname{ad} x$ for some $x \in L_{\infty}^{n}$. Evidently $I+\operatorname{ad} x$ is also an automorphism of $L$. Then since $I+\operatorname{ad} x$ leaves $L_{\infty}^{n}$ invariant and since $K_{1}$ and $K_{2}$ are the unique complements of $L_{\infty}^{n}$ containing $H_{1}$ and $H_{2}$ respectively, $K_{1}$ and $K_{2}$ are conjugate under $I+\operatorname{ad} x$.

We record the following result which has been shown in the proof of Theorem 8 .

Corollary. Let $L_{\infty}^{n}, H$ and $K$ be as in Theorem 8 . Then $H$ is contained in a unique complement of $L_{\infty}^{n}$ in $L$ and it is the Fitting null component of $H$ acting on $L$. Furthermore $K$ contains exactly one Cartan subalgebra of $L_{\infty}^{n-1}$ and it is of the form $K \cap L_{\infty}^{n-1}$.

\section{REFERENCES}

1. D. Barnes, Nilpotency of Lie algebras, Math. Z. 79 (1962), 237-238. MR 27 \#180.

2. - On Cartan subalgebras of Lie algebras, Math. Z. 101 (1967), 350-355. MR 36 \#3837.

3. S. Bauman, A note on cover and avoidance properties in solvable groups, Proc. Amer. Math. Soc. 21 (1969), 173-174.

4. R. Carter, On a class of finite soluble groups, Proc. London Math. Soc. (3) 9 (1959), 623-640. MR 22 \#5677.

5. - Nilpotent self-normalizing subgroups of soluble groups, Math. Z. 75 (1960/61), 136-139. MR 23 \#A928.

6. - Nilpotent self-normalizing subgroups and system normalizers, Proc. London Math. Soc. (3) 12 (1962), 535-563. MR 25 \#3988.

7. R. Carter and T. Hawkes, The F-normalizers of a finite soluble group, J. Algebra 5 (1967), 175-202. MR 34 \#5914.

8. P. Hall, On the system normalizers of a soluble group, Proc. London Math. Soc. 43 (1937), 507-528.

9. B. Huppert, Endliche Gruppen. I, Springer-Verlag, Berlin and New York, 1967. MR 37 \#302.

10. G. Seligman, Modular Lie algebras, Springer-Verlag, Berlin and New York, 1967. MR 39 \#6933.

11. R. Carter, Splitting properties of soluble groups, J. London Math. Soc. 36 (1961), 89-94. MR 24 \#A3202.

North Carolina State University, Raleigh, North Carolina 27607 